\title{
Effect of Acute Exposure of Belladonna Mother Tincture on Zebrafish Embryonic Development
}

\author{
MAHIMA SHARMA, V. N. K. GODLAVETI 1 , S. PRAJAPATI², A. KUMAR ${ }^{3}$, A. TRIPATHI, AND P. GUPTA* \\ Department of Pharmacology, Dr. D. P. Rastogi Central Research Institute of Homeopathy, Noida, Uttar Pradesh 201301, \\ ${ }^{1}$ Department of Biology, Central Forensic Science Laboratory, Ministry of Home Affairs, Kharadi, Maharashtra 411014, \\ 2Department of Pharmacology, Institute of Nuclear Medicine and Allied Sciences, New Delhi 110054, ${ }^{3}$ Department of \\ Pharmacology, Dr. Anjali Chatterjee Regional Research Institute For Homoeopathy, Kolkata, West Bengal 700035, India
}

\section{Sharma et al.: Effect of Belladonna on Zebrafish Embryos}

\begin{abstract}
Atropa belladonna is a highly poisonous plant which contains variety of active alkaloids such as atropine, scopolamine and many others. In homeopathy, its preparation is used to cure a number of health conditions such as baby teething, pain or infection and many others. However, due to its toxic nature and adverse teratogenic effect, it is important to investigate the possible toxicity and safety level of belladonna mother tincture. In this study, zebrafish embryos were used to evaluate fetal embryo toxicity of the belladonna mother tincture. Briefly, eggs were exposed to different concentration $(1,5$ and $10 \%)$ of belladonna mother tincture and ethyl alcohol for $1 \mathrm{~h}$. Different parameters like mortality, hatching rate, somites, tail detachment and morphological malformations were recorded till $72 \mathrm{~h}$ of post fertilisation and behaviour parameters were also recorded after $7 \mathrm{~d}$ post fertilisation. Results showed that exposure to belladonna mother tincture at higher concentration $(5,10 \%)$ cause embryonic malformation including pericardial edema, increase in body length, eye width and heart rate, while decrease hatching rate with no effect on mortality. The behavioural parameters revealed that belladonna mother tincture increases the locomotor activity of larvae at its $1 \%$ concentration rather than 5 and $10 \%$ in comparison to control. In summary, belladonna mother tincture and alcohol may cause developmental embryonic changes at higher concentration while no major development changes were found at $1 \%$. Further developmental toxicity studies on animals need to be carried out to validate use of belladonna mother tincture clinically in low concentration or high dilution.s.
\end{abstract}

Key words: Danio rerio, embryo, developmental toxicity, homeopathy, malformation

The application of natural drugs is increasing day by day in every field and recent scientific community have been focused for drugs from natural sources in drug discovery due to their meagre side effects. Homoeopathy is one of the alternative medicinal systems used globally, which employs drugs prepared from natural origin for the management of diseases. Due to low or negligible concentration of the chemical constituents at higher dilutions/potencies, homoeopathic drugs are generally found to be safe. Now a days, for scientific validation and global acceptance of homoeopathic drugs, in vitro as well as in vivo pharmacological evaluation of these drugs has been greatly increased ${ }^{[1]}$. Since origin of most of the homoeopathic drugs are plant based and raw materials for many homeopathic drugs are known to be toxic, the safety evaluation should not be ignored specifically when using low concentrations of a toxic starting material.

*Address for correspondence

E-mail: pankajgupta77@gmail.com

September-October 2021
Belladonna is one of the most popular homoeopathic drugs used in baby teething, sudden severe pain and pain with inflammation or infection ${ }^{[2]}$. It is prepared from plant Atropa belladonna, commonly known as 'deadly nightshade' known to be most poisonous plant in world ${ }^{[3]}$. Belladonna was the subject of the first homeopathy publication in the Journal Lancet in $1829^{*}$ on its prophylactic and therapeutic efficacy for scarlet fever ${ }^{[4]}$. It is used in traditional treatments for an assortment of conditions including headache, menstrual symptoms, peptic ulcer, histaminic reactions,

\footnotetext{
This is an open access article distributed under the terms of the Creative Commons Attribution-NonCommercial-ShareAlike 3.0 License, which allows others to remix, tweak, and build upon the work non-commercially, as long as the author is credited and the new creations are licensed under the identical terms
}

Accepted 18 September 2021

Revised 30 July 2021

Received 24 February 2020 Indian J Pharm Sci 2021;83(5):947-954 
inflammation and motion sickness, used to overcome bronchial spasms, whooping cough, Parkinson's disease, antidote for snakebite, gastric agent. It contain up to 20 different tropane alkaloids including atropine, scopolamine and hyoscyamine that function as the plant's chemical defence in conditions of stress ${ }^{[5]}$. These alkaloids showed anti-cholinergic toxicity and can cause nervous system inhibition and overdose may cause hallucinations, leading to respiratory arrest and death. It has been reported that atropine and scopolamine can cross the placental barrier and also accumulate in breast milk; however their correlation with genotoxicity, teratogenecity is not known. There are number of cases reported regarding the teratogenic effect of belladonna when taken by pregnant women. Although one cohort study revealed that there was no correlation with fetal malformation and belladonna ${ }^{[6]}$. Teratogen Information System (TERIS) reported that atropine is not significant teratogen; however, atropine has been demonstrated to affect the heart rate of foetus upon exposure during development ${ }^{[7]}$. Though, belladonna mother tincture $(\phi)$ in homeopathic system is not a choice of drug due to its toxic nature but, its higher potencies are the choice of medication in many clinical conditions ${ }^{[8]}$. Few studies reported the use of belladonna in case of certain pregnancy, parturition and lactation, belladonna $\phi$ in some specific conditions and in overdose toxicity ${ }^{[9]}$. However; no preclinical scientific data is available till date in homoeopathy about the toxicity of the belladonna $\phi$.

Rodents are the preferred choice of toxicity evaluation of any drug and usages of these species has been increased tremendously in recent time ${ }^{[10]}$. However, uses of rodents possess substantial limitations, principally with respect to cost, time, ethical approvals and sample size ${ }^{[11]}$. Hence, Zebrafish (Danio rerio) has become a prominent vertebrate model for assessing toxicity, efficacy and safety pharmacological evaluation of number of drugs, because of small size, transparency, higher breeding capabilities, rapid development and genetic similarities between zebrafish and humans ${ }^{[12-14]}$. The National Toxicology Program of the United States of America is also working on establishing and validating of zebrafish as a model for rapid toxicological assessment for pharmaceuticals ${ }^{[15]}$. Hence, the present study was aimed to investigate the effects on development of zebrafish embryos exposed to belladonna $\phi$ at different concentration for $1 \mathrm{~h}$ and assessed the sequence of assessments, including embryonic mortality, hatching rate, presence of malformations and behavioural observations.

\section{MATERIALS AND METHODS}

\section{Drug:}

$\phi$ of Belladonna was procured from the Homeopathic pharmacy industry (GMP Certified Pharma Industry). Alcohol and Belladonna $\phi$ were diluted to 1, 5 and $10 \%$ concentrations using medium used for embryo development (embryo medium; $5 \mathrm{mmol} / \mathrm{l}$ sodium chloride $(\mathrm{NaCl}), 0.33 \mathrm{mmol} / \mathrm{l}$ calcium chloride $\left(\mathrm{CaCl}_{2}\right), 0.33 \mathrm{mmol} / 1$ magnesium sulfate heptahydrate $\left(\mathrm{MgSO}_{4} .7 \mathrm{H}_{2} \mathrm{O}\right), 0.17 \mathrm{mmol} / \mathrm{l}$ potassium chloride $\left.(\mathrm{KCl})\right)$ [16].

\section{Zebrafish husbandry and management:}

Zebrafish were housed in groups (10 Zebrafish/5 1 tank) in clean poly-sulphone tanks filled with filtered, charcoal and UV treated water. Zebrafish were maintained in a controlled environment with a temperature at $27-29^{\circ}$ and a light/dark cycle of $\sim 14 / 10 \mathrm{~h}$ respectively. For all experiments the $\mathrm{pH}$ of the water was maintained between $\mathrm{pH}=6.8-7.5$ with constant filtration and aeration. Water conditioning and environmental quality was maintained according to the aquarium system use, care manual and The Zebrafish Book ${ }^{[16]}$. Zebrafish were fed with dry brine shrimp tetra bits diet thrice a day.

\section{Zebrafish breeding and collection of eggs:}

Male and female zebrafish (at a ratio of 1:2) in a breeding group were placed in tanks few hours before the onset of darkness on the day prior to the test. Spawn traps were placed into the tanks to collect the eggs. Artificial plants made of inert material (plastic or glass) were fixed to the mesh as spawning stimulus. On next morning, the lights were switched on. Mating, spawning and fertilisation took place within $45 \mathrm{~min}$ after the onset of light. The fish were returned to their home tank and the spawn traps with the collected eggs were carefully removed and the eggs were placed in the petri dishes containing embryo medium. Only healthy (no irregularities like asymmetry, vesicle formation, injuries of the chorion) eggs were selected for the study by using an inverted microscope (RTC-7, Radical scientific equipments Pvt. Ltd., Ambala, India).

\section{Grouping:}

Fertilised eggs were divided randomly into seven groups namely; Water (Control), Alcohol (Alc) $1 \%$, Alc $5 \%$, Alc $10 \%$, Belladonna $\phi$ (Bella.) $1 \%$, Bella. $5 \%$ and Bella. $10 \%$ (test solutions) and each group contain ten eggs in 24 well plates for further experiment. A pilot 
study carried out at our facility, alcohol at more than $10 \%$ was found to be lethal to embryos. Therefore, to minimize the toxic effect of vehicle, the concentration of alcohol and test drug was kept $10 \%$ or less for this study ${ }^{[17]}$.

\section{Brief procedure:}

For the purpose of Zebrafish Embryo Toxicity Test (FET), the procedures were adopted from several standard methods ${ }^{[18]}$. The adopted protocol of the study was approved by Institutional Animal Ethics Committee (IAEC), Dr. D. P. Rastogi Central Research Institute of Homeopathy (DDPR-CRIH/Pharmacology/CPCSEA/ IAEC/2018/005). The test was initiated immediately after fertilisation of the eggs and terminated after $7 \mathrm{~d}$ post fertilisation. To start exposure with minimum delay, fertilized randomized viable eggs $(n=10$ per dose group) were transferred to 24 -well plates preconditioned for $24 \mathrm{~h}$ with test solutions and refilled with $2 \mathrm{ml} /$ well freshly prepared test solutions. Then embryos were exposed for a period of $1 \mathrm{~h}$ starting from $3 \mathrm{~h}$ of post fertilisation. $1 \mathrm{~h}$ i.e. 3-4 h of post fertilisation of embryos which corresponds to initial blastula period of embryonic development of mammals. In zebra fish, Mid Blastula Transition (MBT) ensues during the tenth cell division, which is approximately $3 \mathrm{~h}$ of post fertilisation. Exposure of any toxic agents after MBT may induce cell cycle arrest and sometimes accompanied by initiation of an apoptotic program ${ }^{[19]}$. In parallel, control (embryo medium) and alcohol groups in same concentration were also exposed. After exposure time, test solutions were removed from well plates and were replaced by embryo water for entire duration of the study. The entire study was replicated 3 times with same protocol.

\section{Observations:}

Observations were recorded on each tested embryo group under the inverted phase contrast microscope to confirm the morphological characteristics. The following parameters were observed by blinded observers periodically: mortality, hatching, coagulation on 24,48 and $72 \mathrm{~h}$ of post fertilisation and heartbeat, pericardial edema, yolk sac edema, length and eyewidth was recorded on $72 \mathrm{~h}$ of post fertilisation. On the $7^{\text {th }} \mathrm{d}$ after fertilisation (168 $\mathrm{h}$ of post fertilisation), the parameters of locomotor activity: total distance travelled and average speed. Briefly, the well plate was divided into the quadrant and the locomotor activity was recorded for 2 min by making protocol with the help of video tracking software (ANY maze software, 5.21).

\section{Statistical analysis:}

All values were expressed as mean \pm standard deviation (SD). All the statistical analysis of data was done using one way Analysis of Variance (ANOVA) followed by Tukey's Honest Significant Difference test to monitor significance among groups. ${ }^{\mathrm{a}} \mathrm{p}<0.05$ was considered as significant.

\section{RESULTS AND DISCUSSION}

Effect of different concentration of belladonna $\phi$ (1, 5 and $10 \%)$ on mortality rate and hatching rate is illustrated in fig. 1(a) and fig. 1(b) respectively. Representative diagram of somite cell formation and detachment of tail at $24 \mathrm{~h}$ of post fertilisation is shown in fig. 1(c) and at $48 \mathrm{~h}$ of post fertilisation is shown in fig. 1(d). The belladonna $\phi$ treated groups did not show any difference in mortality rate as compared to control group at any time point. Whereas considerable mortality was found with Alc. $10 \%(30 \%, 40 \%$ increased at 48 and $72 \mathrm{~h}$ of post fertilisation respectively) group as compared to control group at $48 \mathrm{~h}$ of post fertilisation and $72 \mathrm{~h}$ of post fertilisation. A remarkable decrease in hatching rate in belladonna $5 \%$ and $10 \%$ at $48 \mathrm{~h}$ of post fertilisation was observed as compared to control group, however it was found to be normal at $72 \mathrm{~h}$ of post fertilisation in all groups. Appearance of Somite with sharp edges was not clear in Alc $5 \%$, Alc $10 \%$ and belladonna $10 \%$ at $24 \mathrm{~h}$ of post fertilisation. Partial tail detachment was recorded in belladonna 5 and $10 \%$ exposure at $48 \mathrm{~h}$ of post fertilisation in zebrafish.

Fig. 2(a) shows the images at $72 \mathrm{~h}$ of post fertilisation, morphological changes; yolk sac edema in fig. 2(b), pericardial edema in fig. 2(c), length in fig. 2(d) and eye width in fig. 2(e) in larvae. Results of fig. 2(b) revealed that yolk sac edema was significantly increased in Alc ( 5 and $10 \%$; ${ }^{\mathrm{a}} \leq 0.05$ ) as compared to control. While belladonna at 1, 5 and $10 \%$ did not show any significant alteration ( $\left.{ }^{\mathrm{a}} \mathrm{p} \geq 0.05\right)$ as compared to control group.

Pericardial edema in zebrafish embryo (fig. 2(c)) had significantly increased with belladonna 1, 5 and $10 \%$ and Alc $10 \%$ group $\left({ }^{\mathrm{a}} \mathrm{p} \leq 0.05\right)$ as compared to control group. In addition, belladonna $1 \%$ showed significant increment $\left({ }^{b} \mathrm{p} \leq 0.05\right)$ in pericardial edema as compared to Alc $1 \%$ group.

As seen in fig. 2(d), the length of the zebrafish larvae had considerably augmented with belladonna $10 \%$ as same 


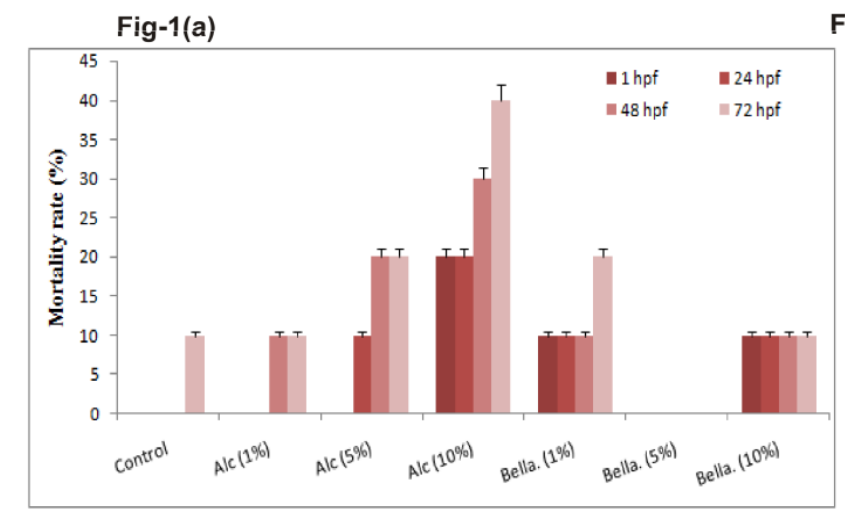

Fig-1(b)

Fig-1@
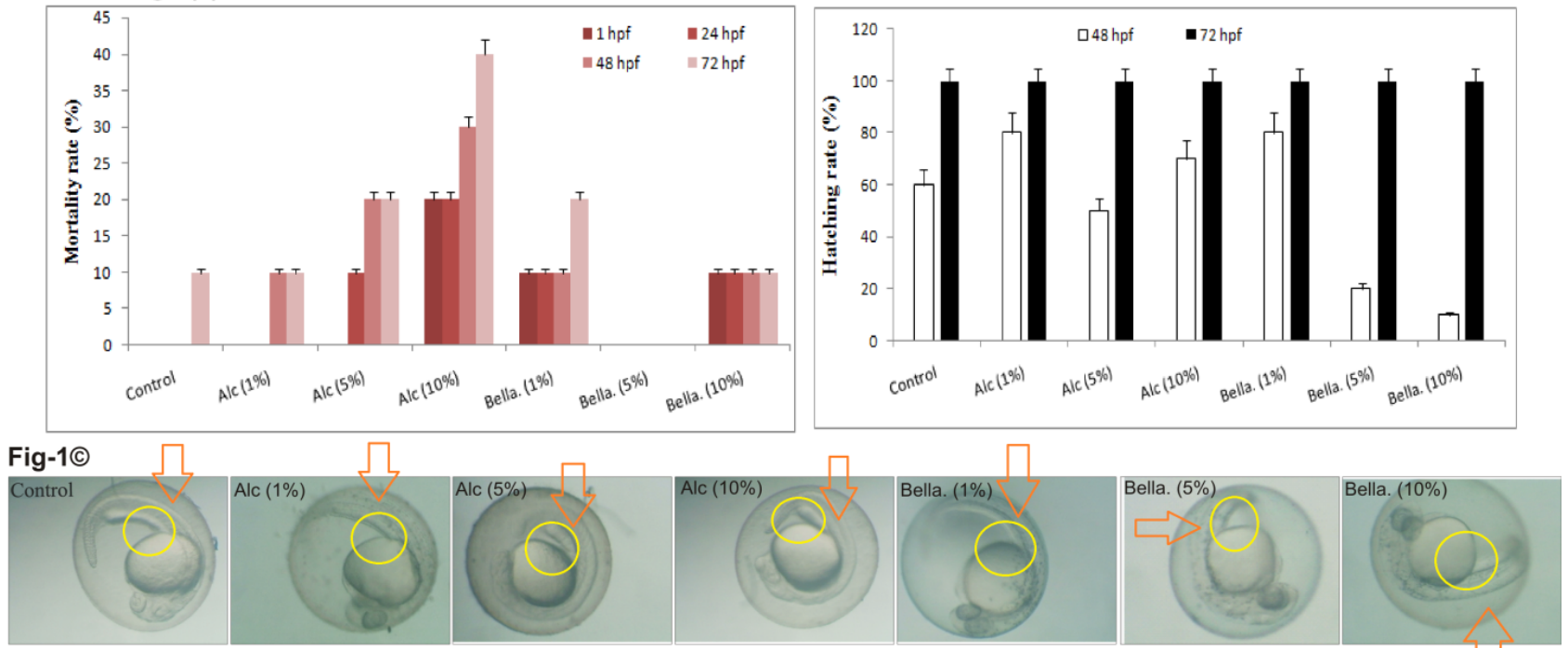

Fig-1(d)

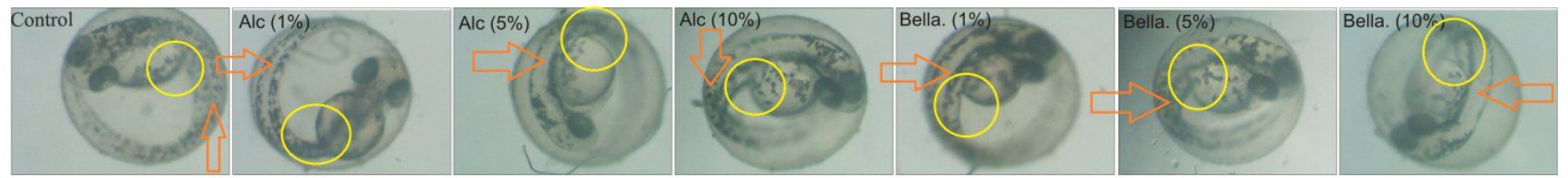

Fig. 1: Effects of $1 \mathrm{~h}$ exposure of belladonna $\phi$ (Bella.; 1, 5 and $10 \%$ ) on (a) percentage of mortality rate; (b) percentage of hatching rate; (c) Representative diagram of somite cell formation and detachment of tail at $24 \mathrm{hpf}$ and (d) At $48 \mathrm{hpf}$
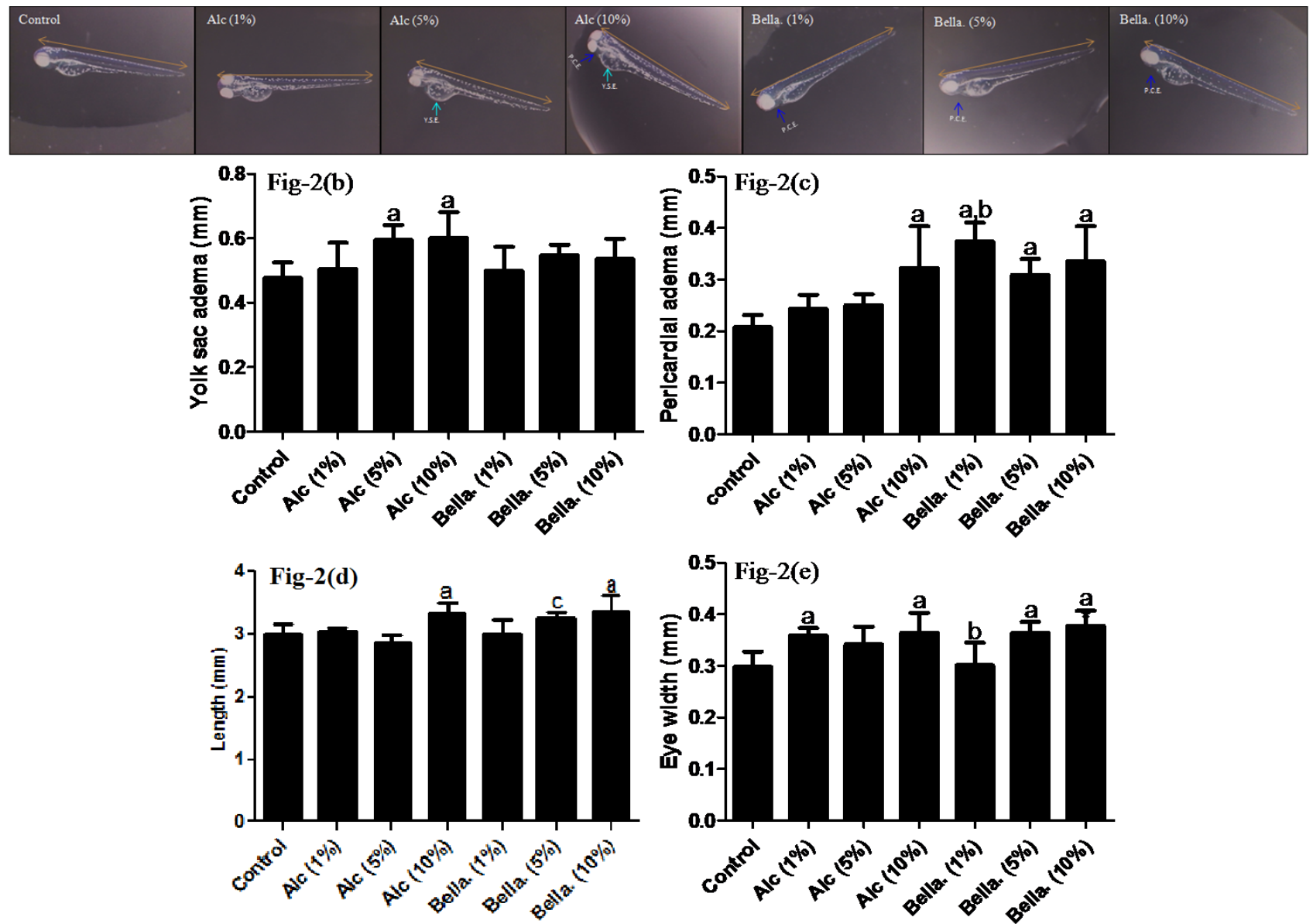

Fig. 2: (a) Larvae images at 72 hpf; (b) effects of $1 \mathrm{~h}$ exposure of belladonna $\Phi$ (Bella.; 1, 5 and $10 \%$ on yolk sac edema; (c) pericardial edema; (d) length and (e) eye width of the zebrafish embryo

Note: All values are mean $\pm S D(n=6),{ }^{a} p \leq 0.05$ compared to control; ${ }^{b} p \leq 0.05$ compared to Alc-1 \%; ${ }^{c} p \leq 0.05$ compared to Alc-5 \% (One way ANOVA followed by Tukey's post hoc test) 
as Alc $10 \%\left({ }^{\mathrm{a}} \mathrm{p} \leq 0.05\right)$ as compared to control. However, no significant changes were observed compared to respective alcohol groups. In addition, belladonna $5 \%$ showed significant increase in length $\left({ }^{c} p \leq 0.05\right)$ as compared to its corresponding alcohol concentration i.e., Alc $5 \%$ group but non-significant as compared to control group. Moreover, Alc $5 \%$ group did not observe any significant changes with control group indicating no profound effect of belladonna $5 \%$ on length.

Statistical analysis revealed that eye-width of zebrafish larvae had significantly increased ( $\left.{ }^{\mathrm{a}} \mathrm{p} \leq 0.05\right)$ with Alc 1 , $10 \%$, belladonna 5 and $10 \%$ when compared to control. Belladonna $1 \%$ showed significant decrease $\left({ }^{b} p \leq 0.05\right)$ as compared to its corresponding alcohol concentration i.e., Alc $1 \%$ while there was no significant sign of alteration in eye width of larvae $\left({ }^{\mathrm{a}} \mathrm{p} \geq 0.05\right)$ as compared to control group as depicted in fig. 2(e).

Effect of belladonna on cardiac function was observed by recording the heartbeats at 48 and $72 \mathrm{~h}$ of post fertilisation (fig. 3). At 48 h of post fertilisation, Bella $10 \%$ along with Alc $5 \%$ and $10 \%$ showed significantly increased $\left({ }^{a} p \leq 0.05\right)$ the heart rate as compared to control group. At $72 \mathrm{~h}$ of post fertilisation the effect of Bella $(1,5$ and $10 \%)$ was not significantly changed $\left({ }^{a} p \geq .05\right)$ as compared to control group only Alc $10 \%$ showed significant increased $\left({ }^{a} \mathrm{p} \leq 0.05\right)$ heart rate as compared to control group.

Effect of different concentration of belladonna on behaviour of zebrafish embryos in term of locomotor activity at $7 \mathrm{~d}$ post fertilisation is demonstrated in fig. 4. Post hoc analysis revealed that the total distance travelled (fig. 4(a) and average speed (fig. 4(b) of the larvae at $7 \mathrm{~d}$ post fertilisation in belladonna $1 \%$ was significantly increased as compared to control $\left({ }^{\mathrm{a}} \mathrm{p} \leq 0.05\right)$ as well as compared to Alc- $1 \%\left({ }^{\mathrm{b}} \mathrm{p} \leq 0.05\right)$. No significant change ( $\left.{ }^{\mathrm{a}} \mathrm{p} \geq 0.05\right)$ observed with belladonna $5,10 \%$ and alcohol groups $(1,5,10 \%)$ when compared to control group.

Zebrafish a well-recognized model became a powerful tool to explore the initial probability of developmental and teratogenic effects of new chemical entities as well as existing medicines. This is now becoming popular method in assessing the effects of herbal medicines on embryonic development including homeopathic medicines ${ }^{[20,21]}$.

Since most of the homoeopathic medicines are also plant based, it is also essential to check the embryonic developmental effects present, if any. Thus, the present study was designed to assess the developmental effects of homoeopathic medicine belladonna $\phi$ in zebrafish embryos. In homeopathy, medicines are commonly prepared in alcohol. It is reported that alcohol is also induce developmental deformities in early phases of life of zebrafish ${ }^{[22]}$. A pilot study carried out at our facility, alcohol at more than $10 \%$ was found to be lethal to embryos as reported in earlier findings ${ }^{[17]}$. Therefore, the concentration of alcohol and test drug was kept $10 \%$ or less for this study. Hence, to minimize the toxic effect of vehicle, alcohol at 1, 5 and $10 \%$ concentrations were tested along with belladonna $\phi$ to rule out the bias of alcohol effects on zebrafish embryos.

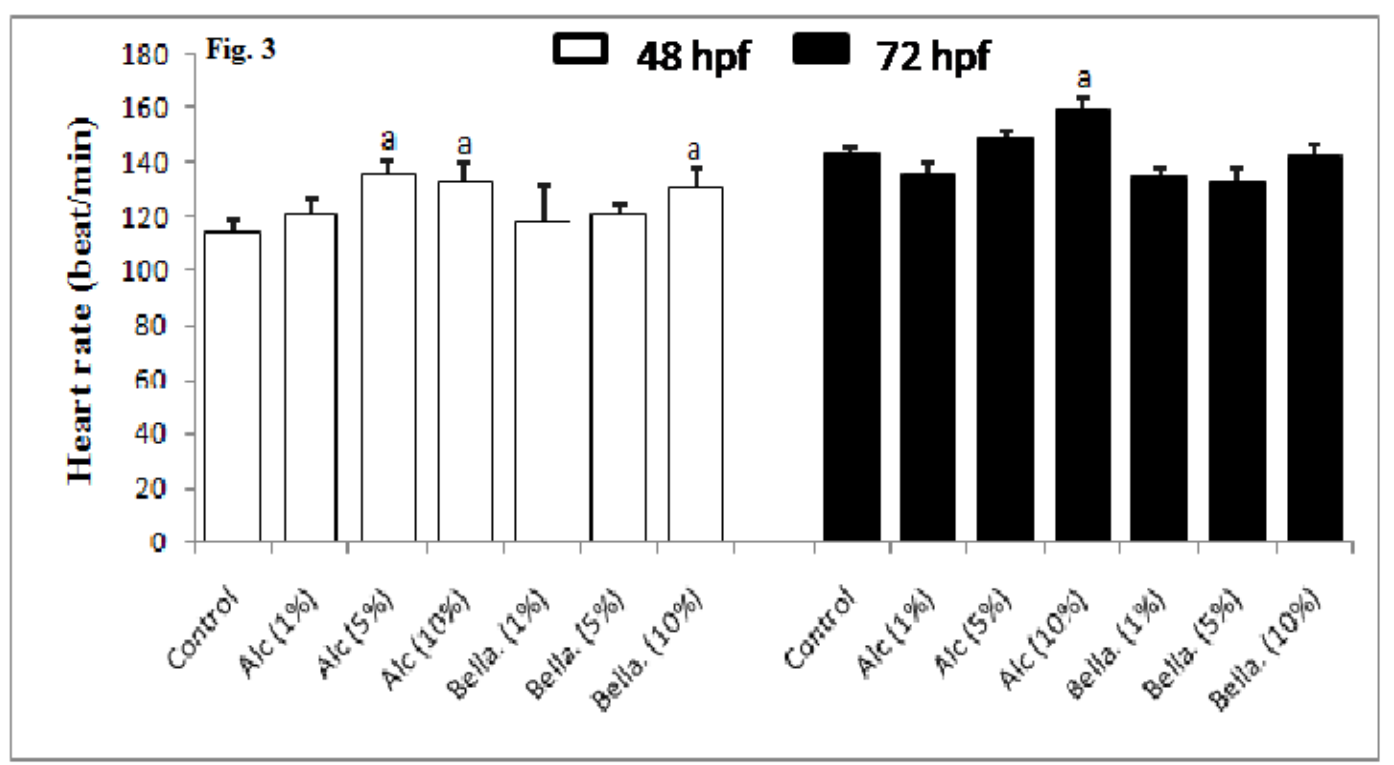

Fig. 3: Effects of $1 \mathrm{~h}$ exposure of belladonna $\phi$ (Bella; 1, 5 and $10 \%$ ) on heart rate of embryos at 48 and 72 hpf Note: All values are mean \pm SD $(n=6),{ }^{a} p \leq 0.05$ compared to control; ${ }^{c} p \leq 0.05$ compared to Alc-5 $\%$ (One way ANOVA followed by Tukey's post hoc test) 

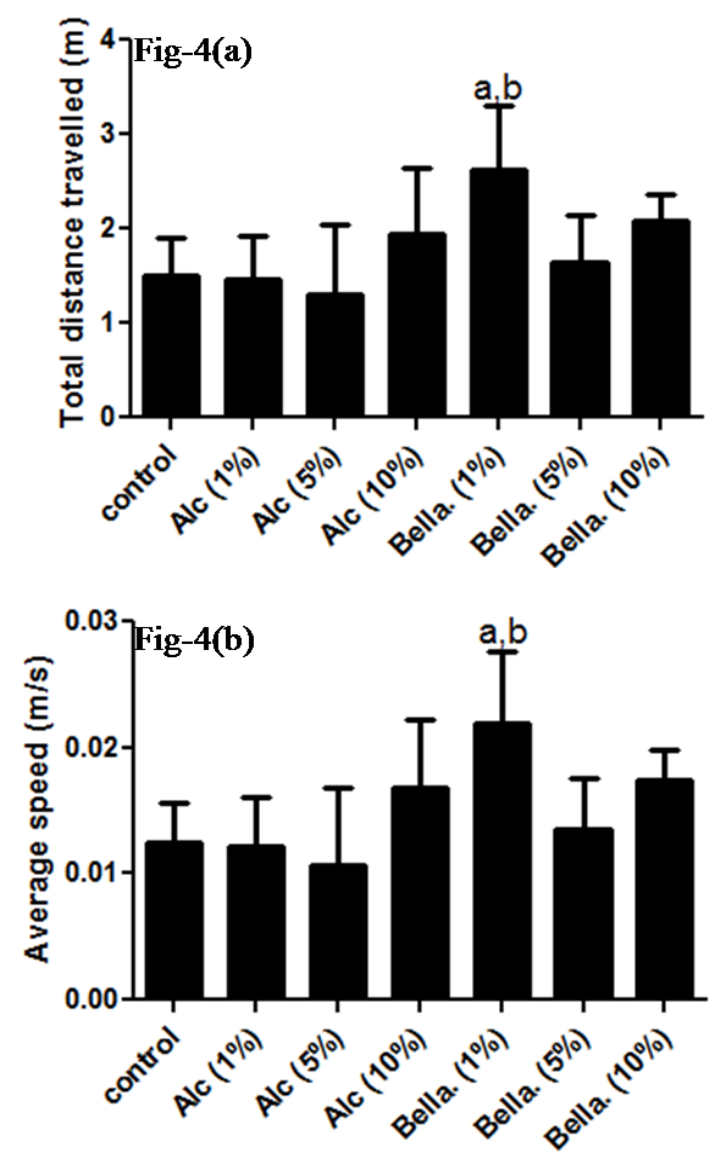

Fig. 4: Effects of $1 \mathrm{~h}$ exposure of belladonna $\phi$ (Bella; 1, 5 and $10 \%$ ) on behaviour of zebrafish larvae at $7 \mathrm{dpf}$ in term of locomotor activity ((a) total distance travelled and (b) average speed)

Note: All values are mean \pm SD $(n=6),{ }^{a} p \leq 0.05$ compared to control; ${ }^{b} \mathrm{p} \leq 0.05$ compared to Alc-1 \% (One way ANOVA followed by Tukey's post hoc test)

In the present study, fertilised viable eggs were exposed to belladonna $\phi$ for a period of $1 \mathrm{~h}$ i.e. $3-4 \mathrm{~h}$ of post fertilisation of embryos which corresponds to initial blastula period of embryonic development of mammals. In zebra fish, MBT ensues during the tenth cell division, which is approximately $3 \mathrm{~h}$ of post fertilisation. MBT is accompanied by loss of division synchrony, increased cell cycle duration, activation of zygotic transcription and an onset of cellular motility in similar to other mammals. Exposure of any toxic agents after MBT may induce cell cycle arrest and sometimes accompanied by initiation of an apoptotic program ${ }^{[19]}$. The developmental effects were evaluated by measuring the presence of any deformities like embryos coagulation, lack of somite cell formation, non-detachment of the tail, lack of heartbeat and yolk sac edema periodically over a period of $72 \mathrm{~h}$. Behavioural observations were made on $7 \mathrm{~d}$ post fertilisation by assessing locomotor activity. These observations were similar in earlier embryonic and teratogenic studies carried out on different chemicals reflecting the predictive power of zebrafish embryo bioassay for embryonic development in mammals.

Under normal conditions, the completion of zebrafish embryogenesis (process by which embryo develop) takes about $72 \mathrm{~h}$ of post fertilisation and for further tissue and organ development, it takes approximately $120 \mathrm{~h}$ of post fertilisation. Coagulation of embryo and lack of heart beat were considered as indicators of mortality during zebra fish embryogenesis as per Organisation for Economic Co-operation and Development (OECD) guideline, $236^{[18]}$. In this study, belladonna $\phi 1,5$ and $10 \%$ showed no changes in mortality rate of zebrafish embryos when compared with control and alcohol (1, 5 and $10 \%)$. However mortality rate was on higher side with Alc $10 \%$ upto $72 \mathrm{~h}$ when compared to control. Thus from the results it is evident that $1 \mathrm{~h}$ exposure of belladonna $\phi$ upto a concentration of $10 \%$ had no lethal effect on zebrafish embryos.

Hatching is known as important process of transformation of embryo to a larva that depends on hatching enzyme activity as well as the movement of embryos and normally, it takes between 48 to $72 \mathrm{~h}$ of post fertilisation to hatch ${ }^{[23]}$. In current study, at $48 \mathrm{~h}$ of post fertilisation in belladonna $\phi$ at $5,10 \%$ showed decreased in hatching rate as compared to control while at $72 \mathrm{~h}$ of post fertilisation hatching rate was found to be equal in all the groups. This change may be due to alteration in hatching enzyme activity and movement of embryos at $48 \mathrm{~h}$ in belladonna group which became normal at $72 \mathrm{~h}$ of post fertilisation. In case of vertebrates, somites provide the first body segments (vertebrae and axial skeletal muscles) during embryogenesis ${ }^{[24]}$ which was not clearly segmented after $1 \mathrm{~h}$ exposure of belladonna $10 \%$ while the detachment of tail sign of further organ development was partial after $1 \mathrm{~h}$ of 5 and $10 \%$ of belladonna. These both are the sigh of delayed morphological development in zebrafish at early life stage.

Morphological developments play an important role in evaluation of early stage developmental effects of many compounds. Zebrafish model has number of advantages for this type of study because of transparency of the eggs that can be observed under microscope for each developmental stage. Inside the chorion before $4 \mathrm{~d}$ post fertilisation, embryo persists the survival and growth by the taking their nutrients from yolk sac ${ }^{[25]}$. A review study reported that measurement of yolk sac was used as the key parameter to evaluate the morphological 
changes with sixty substances exposure ${ }^{[26]}$. In present study, no significant changes were observed in yolk sac diameter in belladonna $\phi$ treated groups. On the other hand, in alcohol 5 and $10 \%$ treated groups significant increase in diameter of yolk sac edema was observed. There may be chances that belladonna $\phi$ diminished the edema effect of 5, $10 \%$ alcohol on yolk sac. Changes in length and Eye-width of larvae in belladonna $\phi$ treated groups at higher concentration compared to control may be due to alcohol alone.

In vertebrates, heart is the first complex organ to develop and function ${ }^{[27]}$. Therefore, there is probability of effect of medicines on heart at developmental stages. In the present study, a heart malformation was observed in terms of pericardial edema as well as heart rate of zebrafish larvae. Belladonna $\phi$ showed significant increase in pericardial edema with all concentrations compared to control whereas belladonna 5 and $10 \%$ showed no significant changes in edema when compared to alcohol 5 and $10 \%$ suggesting that the effect observed may be due to alcohol alone or due to synergistic action of belladonna and alcohol.

At $48 \mathrm{~h}$ of post fertilisation, Bella $10 \%$ along with Alc. $5 \%$ and $10 \%$ showed significantly increased $\left({ }^{\mathrm{a}} \mathrm{p} \leq 0.05\right)$ the heart rate as compared to control group. At $72 \mathrm{~h}$ of post fertilisation the effect of Bella 1, 5 and $10 \%$ was not significantly changed $\left({ }^{\mathrm{a}} \mathrm{p} \geq 0.05\right)$ as compared to control group only Alc. $10 \%$ showed significant increased $\left({ }^{a} \mathrm{p} \leq 0.05\right)$ heart rate as compared to control group.

At $48 \mathrm{~h}$, the increased effect of Bella at $10 \%$ may be due to the alcohol effect as both showed same effect. Whereas, at $72 \mathrm{~h}$ of post fertilisation, the heart rate was found normal as control. It may be due to diminishing effect of belladonna on alcohol as the time passes in early life stages of development which needs to be further studied.

For measuring behavioural effects of any compound or medicine that may cause abnormal or dysfunctional behaviour, locomotor activity is found as quantitative endpoint in Danio rerio ${ }^{[28]}$. Swimming pattern is one of the important parts of behavioural research mainly swimming speed when model used is an aquatic organism. Linde et al. reported the effects of homeopathic medicines which raw material present as toxic in nature ${ }^{[29]}$. The zebrafish model is a vertebrate model that is gaining attention for preclinical drug toxicity and drug screening applications. In zebrafish embryos, cardiovascular, nervous and digestive systems develop in less than $1 \mathrm{w}$. Therefore their early life stages are most prone to the adverse effects of any external substances ${ }^{[30,31]}$.

In present study, changes in locomotor activity in terms of total distance travelled and average speed indicates belladonna may have some excitatory effect on lower concentration on Central Nervous System (CNS) which needs to be further validated. The overall effect of belladonna $\phi$ on zebrafish embryo development is depicted in Table 1.

In summary, it may be concluded that belladonna mother tincture showed no mortality in zebrafish embryos. At higher concentration, some changes in development on early life stage of zebrafish embryos was observed like increase in eye width, larvae length, pericardial edema and heart rate at $48 \mathrm{~h}$ on zebrafish embryos. At low concentration (1\%) no major changes were observed. Further developmental toxicity studies on higher animals need to be carried out to validate use of belladonna $\phi$ clinically in low concentration or high dilution.

TABLE 1: EFFECTS OF BELLADONNA $\$$ AND ALCOHOL AT 1, 5, $10 \%$ CONCENTRATIONS ON ZEBRA FISH EMBRYOS WHEN COMPARED WITH CONTROL

\begin{tabular}{|c|c|c|c|c|c|c|c|}
\hline \multirow{2}{*}{ S. No } & \multirow{2}{*}{ Effect } & \multicolumn{3}{|c|}{ Belladonna $\phi$} & \multicolumn{3}{|c|}{ Alcohol } \\
\hline & & $1 \%$ & $5 \%$ & $10 \%$ & $1 \%$ & $5 \%$ & $10 \%$ \\
\hline 1 & Mortality & - & - & - & - & - & $\uparrow$ \\
\hline 2 & Hatching Rate at $48 \mathrm{hpf}$ & - & $\downarrow$ & $\downarrow$ & - & - & - \\
\hline 3 & Hatching Rate at $72 \mathrm{hpf}$ & - & - & - & - & - & - \\
\hline 4 & Yolk Sac Edema & NSD & NSD & NSD & NSD & $S I \uparrow^{\mathrm{a}}$ & $S I \uparrow^{a}$ \\
\hline 5 & Pericardial Edema & SI $\uparrow^{\mathrm{a}, \mathrm{b}}$ & $\mathrm{SI} \uparrow^{\mathrm{a}}$ & SI $\uparrow^{a}$ & NSD & NSD & SI $\uparrow^{\mathrm{a}}$ \\
\hline 6 & Larvae Length & $\mathrm{SI} \uparrow^{\mathrm{a}}$ & $\mathrm{SI} \uparrow^{c}$ & SI $\uparrow^{a}$ & NSD & NSD & NSD \\
\hline 7 & Eye Width & $S D \downarrow^{b}$ & $\mathrm{SI} \uparrow^{\mathrm{a}}$ & $\mathrm{SI} \uparrow^{\mathrm{a}}$ & SI $\uparrow^{\mathrm{a}}$ & NSD & SI $\uparrow^{\mathrm{a}}$ \\
\hline 8 & Heart Rate at $72 \mathrm{hpf}$ & $\mathrm{SI} \uparrow^{\mathrm{a}}$ & SI $\uparrow^{a, c}$ & SI $\uparrow^{a}$ & SI $\uparrow^{\mathrm{a}}$ & $\mathrm{SI} \uparrow^{\mathrm{a}}$ & SI $\uparrow^{\mathrm{a}}$ \\
\hline 9 & $\begin{array}{c}\text { Locomotor Activity (Total distance travelled \& } \\
\text { Average speed) }\end{array}$ & SI $\uparrow^{a b}$ & NSD & NSD & NSD & NSD & NSD \\
\hline
\end{tabular}

Note: $(-)=$ No difference; NSD=No significant difference; SD=Significant decrease; SI=Significant increase; $(\downarrow)=$ Decrease; $(\uparrow)=$ Increase; ${ }^{(a)}=$ Compared to control; $\left({ }^{b}\right)=$ Compared to Alc. $1 \%$ and $\left({ }^{c}\right)=$ Compared to Alc. $5 \%$ 


\section{Acknowledgements:}

We are thankful to Dr. Premkumar Indracanti, Scientist, INMAS-DRDO for providing microscopic facility. We also extend our thanks to Officer In charge, DDPRCRIH, Renu Arya and Dr. Anil Khurana, Director General, CCRH for providing administrative support.

\section{Conflicts of Interest:}

The authors declared no conflicts of interest.

\section{REFERENCES}

1. Nefedchenko AV, Glotova TI, Glotov AG. In vitro and in vivo activity of homeopathic drugs against bovine herpesvirus- 1 and bovine viral diarrhoea virus. Bulg J Vet Med 2015;18(1):56-64.

2. Abbasi J. Amid reports of infant deaths, FTC cracks down on homeopathy while FDA investigates. JAMA 2017;317(8):793-5.

3. Lackovic Z. "Bunanje": XX Century Abuse of Atropa belladonna Halucinogenic Berries in Continental Croatia. Psychiatr Danub 2017;29(3):379-82.

4. Hufeland M. On the prophylactic powers of belladonna against scarlet fever. Lancet 1829;1:135.

5. Wink M, Roberts MF. Compartmentation of alkaloid synthesis, transport and storage. Alkaloids 1998:239-62.

6. Goldman AS. Birth defects and drugs in pregnancy. Am J Hum Genet 1977; 29(5):546-8.

7. Friedman JM, Little BB, Brent RL, Cordero JF, Hanson JW, Shepard TH. Potential human teratogenicity of frequently prescribed drugs. Obstet Gynecol 1990;75(4):594-9.

8. Richard H. A Manual of Pharmacodynamics. J. E. Adlard, printer, Bartholomew Close, E.C. 1867, Part-1, 136-51.

9. Allen TF. The encyclopedia of pure material medica. Vol. 12. B. Jain Publishers; 2011. p. 67-70.

10. Farghali H, KgalaleloKemelo M, Wojnarova L, Canova, NK. In vitro and in vivo experimental hepatotoxic models in liver research: applications to the assessment of potential hepatoprotective drugs. Physiol Res 2016;65(4):417-25.

11. Caballero MV, Candiracci M. Zebrafish as screening model for detecting toxicity and drugs efficacy. J Unexplored Med Data 2018; 3:4.

12. Fako VE, Furgeson DY. Zebrafish as a correlative and predictive model for assessing biomaterial nanotoxicity. Adv Drug Deliv Rev 2009;61(6):478-86.

13. Santoro MM. Zebrafish as a model to explore cell metabolism. Trends Endocrinol Metab 2014;25(10):546-54.

14. Brannen KC, Charlap JH, Lewis EM. Zebrafish teratogenicity testing. Methods Mol Biol 2013;947:383-401.

15. https://ntp.niehs.nih.gov;https://www.fda.gov/about-fda/ office-hief.../national-center-toxicological-research
16. Westerfield M. The Zebrafish book. A guide for the laboratory use of Zebrafish (Danio rerio). $3^{\text {rd }}$ ed. Eugene, OR, University of Oregon Press; 1995. p. 385.

17. Shaukat A, Danielle LC, Alia A, Michael KR. Large-scale analysis of acute ethanol exposure in zebrafish development: A critical time window and resilience. PLoS One 2011;6(5):e20037.

18. OECD guidelines for the testing of chemicals 236: Fish Embryo Acute Toxicity (FET) Test, WHO; 2013.

19. Dekens MP, Pelegri FJ, Maischein HM, Nusslein-Volhard C. The maternal-effect gene futile cycle is essential for pronuclear congression and mitotic spindle assembly in the zebrafish zygote. Development 2003;130(17):3907-16.

20. Le Q, Suzhen Q, Fangjie C, Jie Z, Changping L, Min S, et al. Effects of penthiopyrad on the development and behaviour of zebrafish in early-life stages. Chemosphere 2019;214:184-94.

21. Lewandowski V, Sary C, Campos EC, de Oliveira CAL, Ribeiro RP, Vargas LD. Homeopathy improves production and hatching probability of zebrafish eggs. Lat Am J Aquat Res 2019;47(4):595-601

22. Bilotta J, Barnett JA, Hancock L, Saszik S. Ethanol exposure alters zebrafish development: a novel model of fetal alcohol syndrome. Neurotoxicol Teratol 2004;26(6):737-43.

23. Hwang P, Chou MY. Zebrafish as an animal model to study ion homeostasis. Pflugers Arch 2013;465:1233-47.

24. Heather LS, Michael JFB, Stephen HD. Somite Development in Zebrafish. Dev Dyn 2000;219:287-303.

25. Anderson JL, Carten JD, Farber SA. Zebrafish lipid metabolism: from mediating early patterning to the metabolism of dietary fat and cholesterol. Methods Cell Biol 2011;101:111-41.

26. Ali S, Aalders J, Richardson MK. Teratological effects of a panel of sixty water-soluble toxicants on zebrafish development. Zebrafish 2014;11:129-41.

27. Wang W, Huang C, Lu Y, Hsin J, Prabhakar V, Cheng C, et al. Heart-targeted overexpression of Nip3a in zebrafish embryos causes abnormal heart development and cardiac dysfunction. Biochem Biophys Res Commun 2006; 347(4):979-87.

28. Velki M, Paolo CD, Nelles J, Seiler TB, Hollert H. Diuron and diazinon alter the behavior of zebrafish embryos and larvae in the absence of acute toxicity. Chemosphere 2017;180:65-76.

29. Linde K, Jonas WB, Melchart D, Worku F, Wagner H, Eitel F. Critical review and meta-analysis of serial agitated dilutions in experimental toxicology. Hum Exp Toxicol 1994;13(7):48192.

30. Makri A, Goveia M, Balbus J, Parkin R. Children's susceptibility to chemicals: a review by developmental stage. J Toxicol Environ Health B Crit Rev 2004;7(6):417-35.

31. Gupta HR, Patil Y, Singh D, Thakur M. Embryonic Zebrafish Model-A Well-Established Method for Rapidly Assessing the Toxicity of Homeopathic Drugs:-Toxicity Evaluation of Homeopathic Drugs Using Zebrafish Embryo Model. J Pharmacopuncture 2016;19(4):319-28. 